\title{
DNA Origami as a Tool to Design Asymmetric Gold Nanostructures
}

\author{
George Amoako ${ }^{1}$, Zhou Ming ${ }^{2}$, Moses Jojo Eghan ${ }^{1} \&$ Samuel Sonko Sackey ${ }^{1}$ \\ ${ }^{1}$ University of Cape Coast, Ghana \\ ${ }^{2}$ Tsinghua University, China \\ Correspondence: George Amoako, University of Cape Coast, Ghana. E-mail: gamoako@ucc.edu.gh
}

Received: September 28, 2017

Accepted: November 22, 2017

Online Published: December 6, 2017

doi:10.5539/jmsr.v7n1p1

URL: https://doi.org/10.5539/jmsr.v7n1p1

\begin{abstract}
DNA origami technology provides a versatile approach for the chemical assembly of gold nanostructures. In this study the bottom-up approach of self-assembly using DNA in the origami process has been successfully applied to arrange five AuNPs asymmetrically. The DNA origami templates were modified to have binding sites that were extended with sticky ends to facilitate the attachment of the AuNPs. With the help of thiol chemistry, the AuNPs which were covered with DNA complementary to the sticky ends introduced on the DNA origami surfaces, we were able to attach the nanoparticles to the designed sites. It was realized that there were slight differences in the designed distances and the determined ones which were accounted for potentially by the deposition of the structures on the grids for imaging. The structures were characterized with gel electrophoresis and TEM. This asymmetric arrangement has the potential of exhibiting plasmonic behavior and circular dichroism when light is incident on the structure.
\end{abstract}

Keywords: DNA origami, folding, gelelectrophoresis, TEM, thiol chemistry, gold nanoparticles

\section{Introduction}

Since the advent of DNA origami in 2006 by Rothemund (2006), a lot of research has been ongoing to functionalize origami structures with nanomaterials to have specific properties in areas such as biology, materials science, physics and computer science. This was after a previous study had demonstrated the formation of programmable assembly of branched DNA junctionsand laid down the foundation for the pursuit and construction of nano-devices using DNA (Seeman, 1982). Some research groups (Mirkin, Letsinger, Mucic, \& Storhoff, 1996; Alivisatos et al., 1996) have used DNA to assemble nanoparticles through hybridization as a bottom-up approach. The specific binding of the bases in DNA is responsible for its self-assembly giving a large amount of control in nanoscale devices assembly.

Rothemund's method (2006) is versatile, robust and significant, and can be used to construct both 2-D and 3-D structures. The DNA origami method uses shorter single-stranded staple DNA sequences to fold a long singlestranded scaffold DNA. For single-layered origami structures the mixture is heated for a few hours to fold while multi-layered structures can take up to days. The DNA origami method can produce both 2-D and 3-D structures of pre-arranged shapes (Rothemund, 2006; Douglas et al., 2009a; Douglas et al., 2009b; Amoako et al., 2013). The approximately 7200 base-scaffold and the numerous staples are mixed together in a suitable buffer during folding and then annealed over a certain temperature range (Rothemund, 2006; Douglas et al., 2009b; Castro et al., 2011). Individual staples can be extended with sticky ends (additional sequences) that protrude on the surfaceand allow the use of DNA origami as a template to assemble nanoparticles. Using the covalent bond between gold and sulfur, functionalized gold nanoparticles with complementary sequences can then hybridize with the sticky ends(Mirkin, Letsinger, Mucic \& Storhoff, 1996; Nuzzo \& Allara, 1983; Dubois \& Nuzzo, 1992; Herne \&Tarlov, 1997; Yang, Yau \& Chan, 1998; Bain \& Whitesides, 1989; Hickman et al., 1992; Mucic, Storhoff, Mirkin, \& Letsinger, 1998; Storhoff et al., 2000; Storhoff, Elghanian, Mucic, Mirkin, \& Letsinger, 1998; Jin, Wu, Li, Mirkin, \& Schatz, 2003; Pearson et al., 2012). This process then attaches the gold nanoparticles onto the origami surface.

Well-structured metal nanoparticles could have potential electrical (Pearson et al., 2012), plasmonic (Kuzyk et al., 2012; Shen et al., 2012), and circular dichroism (CD)(Pearson et al., 2012; Shen et al., 2012) characteristics.

In this work, we demonstrate the use of DNA origami to arrange gold nanoparticles (AuNPs) asymmetrically. We believe this asymmetric arrangement of AuNPs has potential applications in plasmonics and circular dichroism. 


\section{Materials, Methods and Design}

\subsection{Materials}

The experimental procedures following were adapted (Castro et al., 2011; Zhao, Jacovetty, Liu, \& Yan, 2011; Tanton, 2002).

\subsubsection{Chemicals and supplies}

All unmodified utrapage purified DNA oligonucleotides and Ethylenediaminetetraacetic acid (EDTA) were purchased from Sangon Biotech (Shanghai) Co. Ltd, suspended in ultrapure water and used without further purification. The 3'- and 5'- thiol-modified HPLC purified DNA strands were obtained from the same source while the Tris (hydroxymethyl) aminomethane (Tris), Agarose $\mathrm{M}$, and magnesium acetate tetrahydrate $\left(\left(\mathrm{CH}_{3} \mathrm{COO}\right)_{2} \mathrm{Mg} \cdot 4 \mathrm{H}_{2} \mathrm{O}\right)$ were obtained from Bio Basic Inc (Markham, Canada). NA-red, and 6X loading buffer were obtained from Beyotime Institute of Biotechnology (Haimen, China) and the wide-range DNA marker from Takara Biotechnology (Dalian, China) Co. Ltd. The single-stranded viral genomic DNA M13mp18 used in the experiments was obtained from New England Biolabs (Ipswich, UK) and the boric acid $\left(\mathrm{H}_{3} \mathrm{BO}_{3}\right)$, magnesium chloride $\left(\mathrm{MgCl}_{2}\right)$, and acetic acid $\left(\mathrm{C}_{2} \mathrm{H}_{4} \mathrm{O}_{2}\right)$ from Sinopharm Chemical Reagent (Shanghai, China) Co. Ltd. Freeze 'N' Squeeze DNA gel-extraction spin columns were acquired from Bio-Rad Laboratories Inc. (Hercules, USA)and Tris (carboxyethyl) phosphine hydrochloride (TCEP) and Bis (p-sulfonatophenyl) phenylphosphinedihydratedipotassium salt (BSPP) from Sigma-Aldrich, USA. 15 and 10nm AuNP colloids wereobtained from Ted Pella Incand other chemical reagents from Sinopharm Chemical Reagent Co. Ltd. Carbon copper grids and mica were bought from Beijing Zhongjingkeyi Technology Co. Ltd and finally uranyl acetate $\left(\mathrm{UO}_{2}\left(\mathrm{CH}_{3} \mathrm{COO}\right)_{2} \cdot 2 \mathrm{H}_{2} \mathrm{O}\right)$ from Structure Probe, Inc. (Beijing, China).

\subsection{Methods}

\subsubsection{Phosphination and Concentration of AuNPs}

We stabilized the $10 \mathrm{~nm}$ AuNPs with absorption of BSPP. BSPP (15 mg) was then added to the colloidal nanoparticle solution $(50 \mathrm{~mL})$ and the mixture shaken overnight with a constant temperature incubator at room temperature. Sodium chloride (solid) was slowly added to the mixture and stirred until the color changed from deep burgundy to light purple. The resulting mixture was centrifuged at $3000 \mathrm{rpm}$ for $30 \mathrm{~min}$ and the supernatant carefully removed with a pipette. AuNPs were re-suspended in $1 \mathrm{~mL}$ solution of BSPP $(0.5 \mathrm{mM}) .500 \mu \mathrm{L}$ methanol was then added to precipitate the particles and the mixture again centrifuged at $3000 \mathrm{rpm}$ for $30 \mathrm{~min}$. The supernatant was carefully removed and the AuNPs re-suspended in $1 \mathrm{~mL}$ of $0.5 \mathrm{mM}$ BSPP. The concentration of the AuNPs was estimated by the optical absorbance at $520 \mathrm{~nm}$. Phosphine coating increases the negative charge on the particle surface and therefore stabilizes the AuNPs in high electrolyte concentrations at high particle density. The whole process was repeated to stabilize the $15 \mathrm{~nm}$ AuNPs.

\subsubsection{Preparation of AuNP-DNA conjugates}

TCEP $(20 \mathrm{mM}, 1 \mathrm{~h})$ in water was used to reduce the disulfide bond in the thiol modified oligonucleotides to monothiol. Size exclusion columns (G-25, GE Healthcare) were used to purify the oligonucleotides and remove smaller molecules. Monothiol modified oligonucleotides and phosphinatedAuNPs were then combined (DNA to AuNP molar ratio of more than 200:1) in 0.5X TBE buffer ( 89 mMTris, $89 \mathrm{mM}$ boric acid, $2 \mathrm{mM}$ EDTA, pH 8.0) containing $50 \mathrm{mMNaCl}$ for 40 hours at room temperature to ensure the AuNPs were fully covered by thiolated DNA. 0.5X TBE buffer in Microcon (100 KDa, Millipore, Billerica, MA) columns were used to wash AuNP-DNA conjugates to remove the excess oligonucleotides. The concentration of these AuNP-DNA conjugates was estimated from the optical absorbance at $520 \mathrm{~nm}$. Freshly prepared AuNPs $(10 \mathrm{~nm}$ and $15 \mathrm{~nm})$, fully covered with DNA strands did not precipitate in buffer (5 mMTris, $1 \mathrm{mM}$ EDTA, $12 \mathrm{mM} \mathrm{MgCl}$ ), which is preferred for the folding in DNA origami.

\subsubsection{Folding and purification of DNA origami cross-like structures}

Our sample was prepared based on the procedures outlined in a previous study (Castro et al., 2011), but with some modifications to the annealing process. This modification involved combining $10 \mathrm{nM}$ scaffold (M13mp18), 100nM of each of the 178 staple oligonucleotides which were used without further purification, buffer and salts including 5 mMTris, 1 mM EDTA ( $\mathrm{PH} 7.9$ at $20^{\circ} \mathrm{C}$ ), and a magnesium screen covering 7 different concentrations from 12 $\mathrm{mM}$ at $2 \mathrm{mM}$ intervals to $24 \mathrm{mM} \mathrm{MgCl}$. Folding was carried out in a PCR tube by rapid heat denaturation followed by slow cooling from $65^{\circ} \mathrm{C}$ to $60^{\circ} \mathrm{C}$ over a 50 minute interval, then $60{ }^{\circ} \mathrm{C}$ to $24{ }^{\circ} \mathrm{C}$ over 72 hours. We performed electrophoresis on samples using 2\% Agarose gel (0.5X Tris/Borate/EDTA (TBE), $11 \mathrm{mM} \mathrm{MgCl}_{2}, 10 \mu \mathrm{L}$ NA-red) at $70 \mathrm{~V}$ for 3.5 hours in an ice-water bath. Discrete bands were visualized with UV trans-illuminator (Peiqing JS- 
680B). The desired bands were physically excised, crunched and filtered through a Freeze 'N' Squeeze spin column at $4{ }^{\circ} \mathrm{C}$ for 10 minutes at $16000 \times \mathrm{g}$.

\subsubsection{Preparation and purification of origami-AuNPs complexes}

The purified DNA origami was mixed with AuNP-DNA conjugates in a ratio of 1:10 and annealed from $40{ }^{\circ} \mathrm{C}$ to room temperature. The annealed product of the DNA origami and AuNPs were loaded in a 1.0\% Gel-red (Biotum) stained agarose gel (running buffer $0.5 \mathrm{X}$ TBE containing $11 \mathrm{mM} \mathrm{MgCl}_{2}$, loading buffer $50 \%$ glycerol, $70 \mathrm{~V}$ constant Voltage). Selected bands were cut out and the DNA Origami-AuNPs complexes extracted from the gel with Freeze'N'Squeeze columns (Bio-Rad Laboratories Inc) at $4{ }^{\circ} \mathrm{C}$.

\subsubsection{TEM imaging}

Transmission electron micrographs were obtained with a JEM-2100 (HR) TEM. The sample was negatively stained. A $3 \mu \mathrm{l}$ sample solution was deposited onto the carbon-coated side of the TEM grid and allowed to adsorb for about 5 minutes. The grid sample-side was first immersed into a $\%$ uranyl acetate (Structure Probe, Inc) stainsolution droplet and incubated for 40 seconds. All excess liquid was dabbed off with the edge of a filter paper, and the grid allowed to dry completely. Images were taken at different accelerating voltages.

\subsection{Design}

\subsubsection{Design of AuNPs Attachment Sites}

The previously reported $12 \times 24 \times 38$ DNA origami cross-like structure was used as the template to organize the AuNP structures (Amoako et al., 2015). The square lattice based caDNAno software was used to design the 3D cross-like structure. The 78 helix bundle multilayer was used to assemble the AuNPs after the following modifications were made to the staple strands at the attachment sites:

We designed a structure with the cross-like DNA origami as template using AuNPs. The idea was to make the nanoparticles structure have an asymmetric shape. We designed five nanoparticles attachment sites with one nanoparticle attachment site at each of the four ends of the cross-like structure and the last attachment site on top of the template. The attachment sites are shown as yellow and blue in Figure 1. Four attachment sites were designed at the bottom of the template with only one on the top surface. We designed the nanoparticles structure such that the separations between opposite sites were not equal. The separation between the attachment site at the top (yellow) and the one directly below (blue) was $16 \mathrm{~nm}$ (Figure 1). The separation between the attachment site on the left (yellow) and the one on the right (blue) was 26 nmwhile the separation between the AuNP on the surface of the template and those at the bottom was $12 \mathrm{~nm}$. The surface nanoparticle was placed approximately at the center of the template.

To attach AuNPs to our cross-like structure, we made modifications to the staple strands at the designed attachment sitesby extending the DNA staples at the designed sites with specific sticky ends. The staples at the blue attachment sites (Figure 1) were extended at the 5' end with sequences ATATATATATATATAA (B) while the staples at the yellow attachment sites (Figure 1) were extended at the 5' end with sequences AAAAAAAAAAAAAAA (C) as shown in Table 1. Each site was designed with three staple sequences so that for the five AuNPs we extended 15 staple strands. The complementary sequences to B and C were N2 and N1 respectively. N1 and N2 were terminated on the 3' end with thiol (-SH) extensions as shown in Table 1 to covalently attach the phosphine stabilized AuNPs. Each of N1 and N2 were 15 ntlong. The two yellow sites were designed to attach two AuNPs and the three blue attachment sites designed to attach three AuNPs. The sticky ends were placed such that after hybridization of the cross-like structure they were displayed on the upper and bottom surfaces of the template.

When the sticky end extensions on the B-labeled sequences in Table 1 hybridize with the complementary sequences N2, the AuNPs stick out on the surface of the template. The sticky end extensions on the C-labeled sequences will also hybridize with the complementary sequences N1 and the AuNPs will stick out on the template surface. 


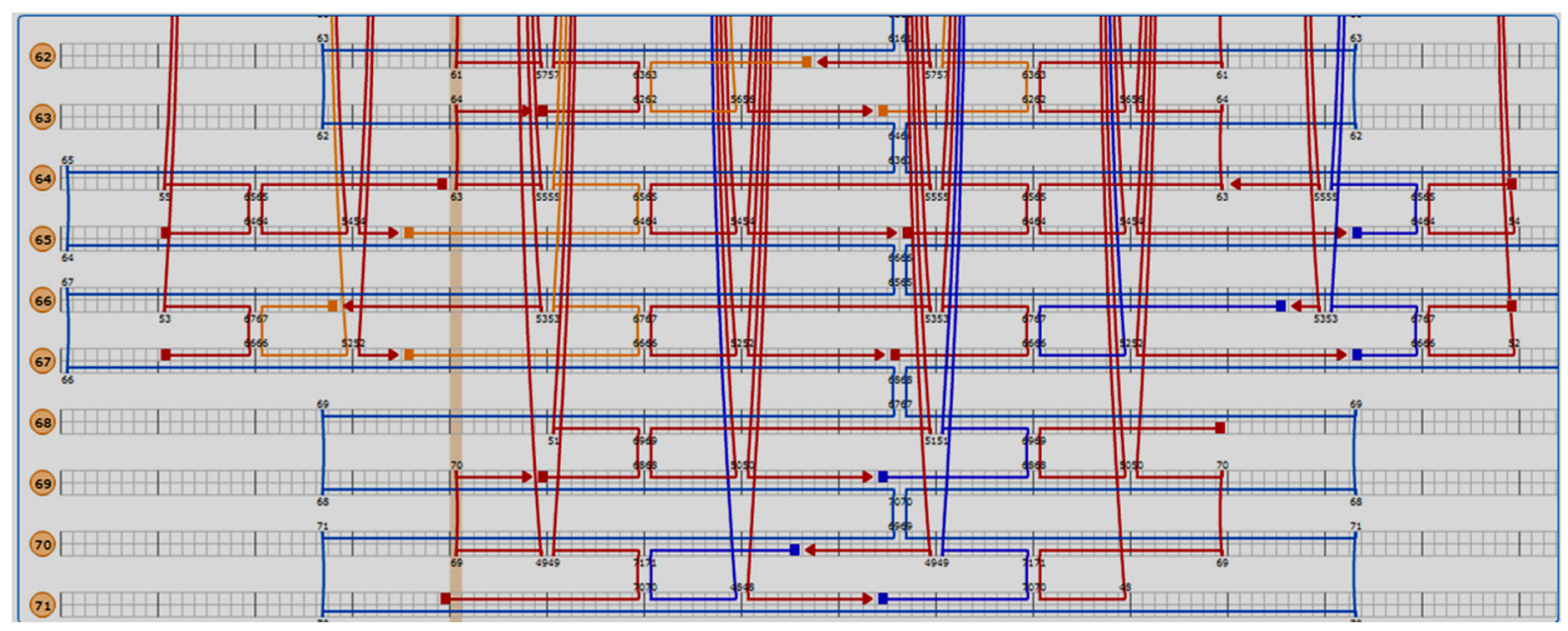

Figure 1. Gold nanoparticles attachment sites. Yellow and blue indicate the designed attachment sites for AuNPs. Red indicates the DNA staple single strands used to fold the scaffold strand (light blue)

Table 1. Staple DNA sequences with the extended sticky ends. B1 to B6 are indicated as yellow in Figure 1while $\mathrm{C} 1$ to $\mathrm{C} 9$ are indicated as blue in Figure 1

\begin{tabular}{lll}
\hline B1 & ATATATATATATATAACAACGAGGGTAGTAAACGGGTAAAATGTAGCGCGTTTT & 54 \\
B2 & ATATATATATATATAGCCTCCCTCAGAGCAACCACCACTTG & 41 \\
B3 & ATATATATATATATAGCCGGTCGCTGAGGACAGCATAGCGATTACTAA & 48 \\
B4 & ATATATATATATATAGAATTTACCGTTCCAGTAAGGCAGGTCAATTCATTAATTTG & 56 \\
B5 & ATATATATATATATAAGTAACAATTTCGGAACGTA & 35 \\
B6 & ATATATATATATATAATTATTCTGAAACATGAAAGAAGTTTACACGG & 48 \\
C1 & AAAAAAAAAAAAAAACGAAAATCCCAGGGTGGTTATCGGGAGAAACACCGGA & 52 \\
C2 & AAAAAAAAAAAAAAATCTTTCACCAGTGAGACGTCCAC & 39 \\
C3 & AAAAAAAAAAAAAAAATTGGGCGGCTAACTCTGTGTTGG & 40 \\
C4 & AAAAAAAAAAAAAAAAGGTGACCGACAATGCGCAGA & 36 \\
C5 & AAAAAAAAAAAAAAAGTTGAAAATCTCCAAAAAAAAACAACTTTGGCTT & 49 \\
C6 & AAAAAAAAAAAAAAAAGAGAATAATTTTTTACCGGATACTGACCAATTAAGA & 51 \\
C7 & AAAAAAAAAAAAAAAAATACAGCCCTCATTTTCTGTAAGTAAATTCTGA & 48 \\
C8 & AAAAAAAAAAAAAAATCAGGAGGTTTAGCCTCATTTCCAATAATAAGAGCAATAATA & 57 \\
C9 & AAAAAAAAAAAAAAAAAAGCCAATAGGAGCATTCCACTCATTATACCTTATGGAGCAA & 58 \\
N1 & TTTTTTTTTTTTT-SH & 15 \\
N2 & TATATATATATATAT-SH & 15 \\
\hline
\end{tabular}

\section{Results and Discussion}

To minimize nonspecific binding between the negatively charged DNA strands and the metal surface, and also to increase the stability of the colloids, we prepared the AuNPs using a negatively charged phosphine shell. This reactive phosphine shell is easily displaced by thiolated DNA strands in the presence of $\mathrm{NaCl}_{2}$. In solution, Brownian motion brings the functionalized AuNPs and the sticky ends on the DNA origami surfaces into close proximity. Since these DNA single strands are complementary they hybridize.

Figure 2 shows the $0.6 \%$ agarose gel electrophoresis of the conjugated DNA origami/AuNP structures. The M13mp18 scaffold DNA strand was used as marker and shown in lane 1. Lane 2 contained the DNA origami cross-like structure and the AuNPs. The AuNP arrangement on the origami structure shown in the Figure indicates the nanoparticles arrangement below the origami structure. The band containing the origami/AuNP complex is as indicated (Figure 2). This lane also contained the excess AuNPs that were not involved in binding, and the band is indicated (Figure 2). 


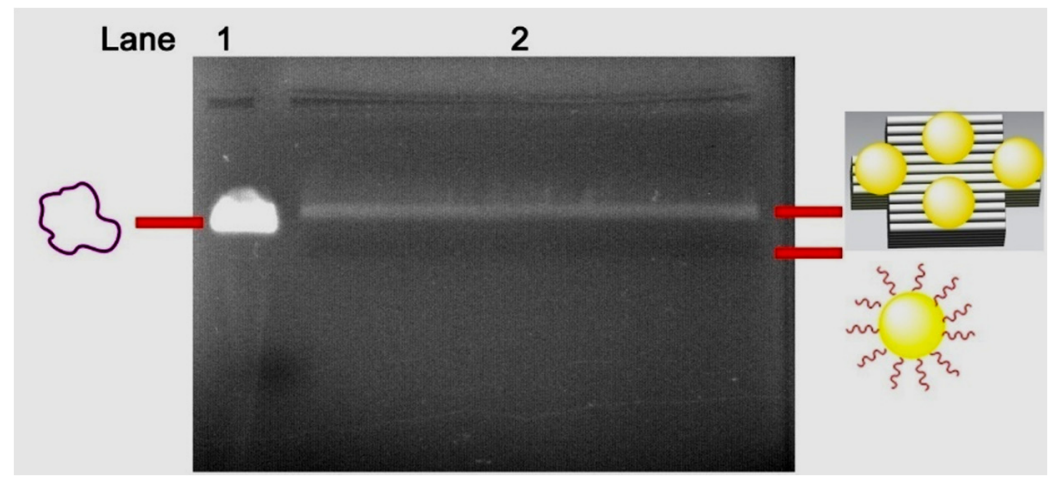

Figure 2. Gel red stained $0.6 \%$ agarose gel electrophoresis of assembled cross-like origami/AuNP products. Lane 1 contains M13mp18 scaffold marker with the band indicated. Lane 2 contains metalized origami structures and the well-formed structure is indicated with the clear band. Excess AuNPs migrate faster and are indicated in the not-clear band

We arranged two types of AuNPs shapes. One type used only the four attachment sites under the DNA origami cross-like structure while the other used all the five designed attachment sites. We used the two types of sticky ends to attach four $15 \mathrm{~nm}$ AuNPs under the cross-like structure as shown in Figure 3. The separation between the AuNPs in Figure 3a match the caDNAno designed separation distances. All the separation distances were determined from the centers of the AuNPs. The separation from the centers of the AuNPs between the left AuNP labeled as 4 (top attachment site in caDNAno in Figure 1) and the right AuNP labeled as 3 (down attachment site in caDNAno in Figure 1) in the TEM image in Figure 3a is $\sim 24 \mathrm{~nm}$. The separation between the top AuNP labeled as 1 (right attachment site in caDNAno in Figure 1) and the down AuNP labeled as 2 (left attachment site in caDNAno in Figure 1) in Figure $3 \mathrm{a}$ is $\sim 40 \mathrm{~nm}$. These two separation distances (that is $1 \rightarrow 2$ and $3 \rightarrow 4$ ) were designed to be $26 \mathrm{~nm}$ and $16 \mathrm{~nm}$ respectively. Figure $3 \mathrm{~b}$ shows that the separation distances are $\sim 30 \mathrm{~nm}$ and $\sim 18$ $\mathrm{nm}$ respectively for $1 \rightarrow 2$ and $3 \rightarrow 4$. In Figure $3 \mathrm{c}$, the separation distances are $\sim 40 \mathrm{~nm}$ and $\sim 20 \mathrm{~nm}$ for $1 \rightarrow 2$ and $3 \rightarrow 4$ respectively.

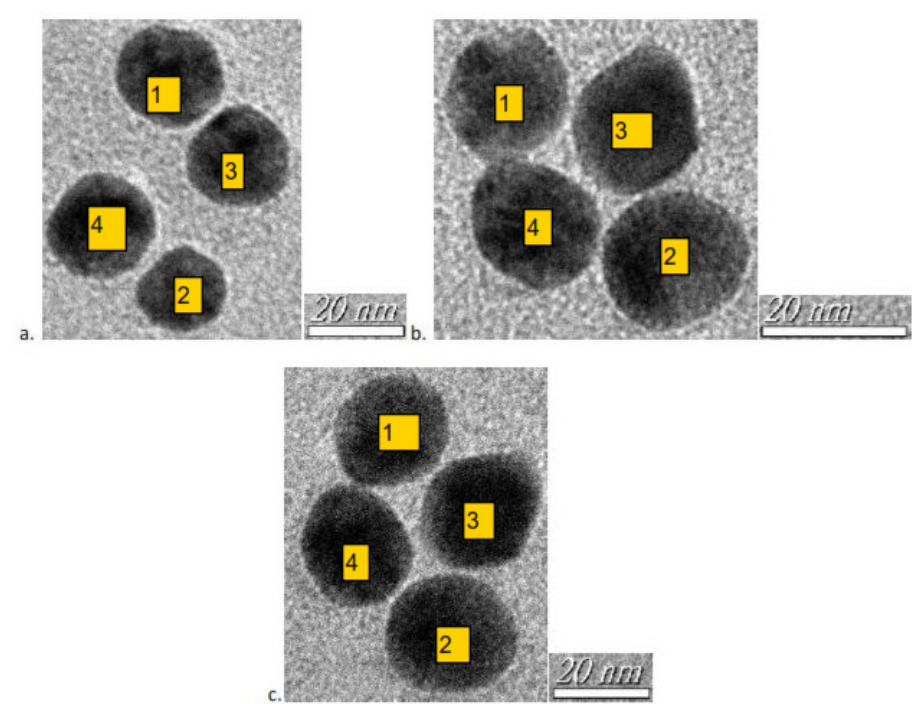

Figure 3. TEM images of $15 \mathrm{~nm}$ AuNP assemblies at the bottom of theDNA origamicross-like structure. (a-c) are cross sections of the four $15 \mathrm{~nm}$ AuNPs structures formed. The scale bars are indicated besides the images

Figure 4 shows the arrangement of 5 AuNPs using all the designed attachment sites on the cross-like structure. Two different diameters of AuNPs were used, and were made up of three $15 \mathrm{~nm}$ and two $10 \mathrm{~nm}$ diameter nanoparticles. The $15 \mathrm{~nm}$ diameter AuNPs are labeled as 2, 4 and 5 in Figure 4 while the $10 \mathrm{~nm}$ diameter AuNPs are labeled as 1 and 3. The TEM image of AuNPs reveals that their shape is not all spherical as was assumed. Some even showed triangular shapes. Figure 3 shows the symmetric nature of the AuNP arrangement. The 
introduction of the fifth AuNP on the surface of the Origami structure breaks this symmetric frame, introduces asymmetry into the arrangement and makes the arrangement three dimensional. The potential coupling of the bottom layer AuNPs to the single AuNP in the upper layer makes the AuNPs structure truly 3-D and possibly chiral. TEM images are used to characterize DNA origami template self-assembly of AuNPs, where the interparticle distance can be used to deduce the designed separation distance. However, increased forces that act on the larger nanoparticles as the sample dries on the TEM grid could be sufficient in distorting the arranged AuNPs shape. Slight deviations from the designed structures could be seen in Figure 4. In Figure 4aand 4b, we see clearly that the number $5 \mathrm{AuNP}$ is placed above number $1 \mathrm{AuNP}$ as designed, but the bottom placed AuNPs are displaced from their designed positions if compared to Figure 3. Figure $4 \mathrm{c}$ shows that the top placed AuNP slightly displaces the number 1 labeled AuNP. The displacement by the number 5 AuNP is complete in Figure $4 \mathrm{~d}$ and therefore moving the number 1 AuNP further away. This displacement renders the AuNP structure in Figure 4d two dimensional.

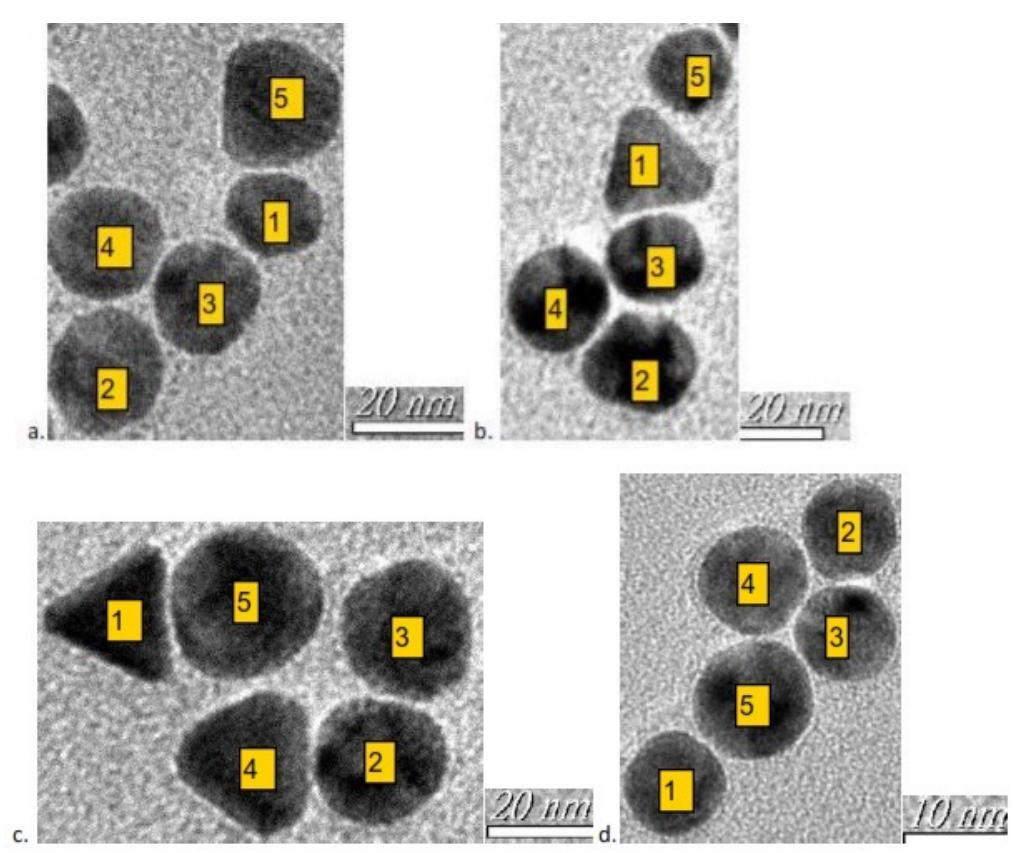

Figure 4. TEM images of $15 \mathrm{~nm}$ and $10 \mathrm{~nm}$ AuNP assemblies using the DNA origami cross-like structure. The scale bars are indicated besides the images

\section{Conclusion}

In conclusion, we have been able to use DNA origami to arrange an asymmetric AuNPs structure with the potential to exhibit plasmonic behavior and circular dichroism. We have also completely characterized our AuNPs structure and determined their inter-particle distances. There were slight differences in the designed distances and the determined distances. These differences were possibly due to the deposition of samples onto the TEM grids for Tem imaging.

\section{Acknowledgment}

This work was supported by the National Basic Research Program of China (973 Program, Grant 2011CB013004) and Major Project of State Key Laboratory of Tribology, Tsinghua University (SKLT10A02).

\section{References}

Alivisatos, A. P., Johnson, K. P., Peng, X., Wilson, T. E., Loweth, C. J., Bruchez Jr., M. P., \& Schultz, P. G. (1996). Organization of 'nanocrystal molecules' using DNA. Nature, 382, 609-611. https://doi.org/10.1038/382609a0

Amoako, G., Zhou, M., Ye, R., Zhuang, L., Yang, X., \& Shen, Z. Y. (2013). 3D DNA origami designed with caDNAno. Chinese Science Bulletin, 58, 3019-3022. https://doi.org/10.1007/s11434-013-5879-y 
Amoako, G., Zhou, M., Ye. R., Mensah-Amoah, P., Twum, A., \& Sam, F. (2015). Connecting DNA origami structures using the biotin-streptavidin specific binding. African Journal of Biotechnology, 14, 2258-2264. https://doi.org/10.5897/AJB2015.14697

Bain, C. D., \& Whitesides, G. M. (1989). Modeling organic surfaces with self-assembled monolayers. Angewandte Chemie International Edition, 28(4), 506-512. https://doi.org/10.1002/anie.198905061

Castro, E. C., Kilchherr, F., Kim, D-N., Shiao, E. L., Wauer, T., Wortmann, P., Bathe, M., \& Dietz, H. (2011). A primer to scaffolded DNA origami. NatureMethods, 8, 221-229. https://doi.org/10.1038/nmeth.1570

Douglas, S. M., Marblestone, A. H., Teerapittayanon, S., Vazquez, A, Church, G. M., \& Shih, W. M. (2009a). Rapid prototyping of 3D DNA-origami shapes with caDNAno. Nucleic Acids Research, 37, 5001-5006. https://doi.org/10.1093/nar/gkp436

Douglas, S. M., Dietz, H., Liedl, T., Hogberg, B., Graf, F., \& Shih, W. M. (2009b). Self-Assembly of DNA into nanoscale three-dimensional shapes. Nature, 459, 414-418. https://doi.org/10.1038/nature08016

Dubois, L. H., \&Nuzzo, R. G. (1992).Synthesis, Structure, and Properties of Model Organic Surfaces. Annual Review of Physical Chemistry, 43, 437-463. https://doi.org/10.1146/annurev.pc.43.100192.002253

Herne, T. M., \& Tarlov, M. J. (1997). Characterization of DNA probes immobilized on gold surfaces. Journal of American Chemical Society, 119, 3401-3402. https://doi.org/10.1021/ja9719586

Hickman, J. J., Laibinis, P. E., Auerbach, D. I, Zou, C., Gardner, T. J., Whitesides, G. M., \&Wrighton, M. S. (1992). Toward orthogonal self assembly of redox active molecules on platinum and gold: selective reaction of disulfide with gold and isocyanide with platinum. Langmuir, 8, 357-359. https://doi.org/10.1021/ la00038a005

Jin, R., Wu, G., Li, Z., Mirkin, C. A., \& Schatz, G. C. (2003). What Controls the Melting Properties of DNALinked Gold Nanoparticle Assemblies? Journal of America Chemical Society, 125, 1643-1654. https://doi.org/10.1021/ja021096v

Kuzyk, A., Schreiber, R., Fan, Z., Pardatscher, G., Roller, E-M., Hoegele, A., Simmel, F. C., Govorov, A. O., \& Liedl, T. (2012).DNA-based self-assembly of chiral plasmonic nanostructures with tailored optical response. Nature, 483, 311-314. https://doi.org/10.1038/nature10889

Mirkin, C. A., Letsinger, R. L., Mucic, R. C., \& Storhoff, J. J. (1996). A DNA-based Method for Rationally Assembling Nanoparticles into Macroscopic Materials. Nature, 382, 607-609. https://doi.org/10.1038/ $382607 \mathrm{a} 0$

Mucic, R. C., Storhoff, J. J., Mirkin, C. A., \& Letsinger, R. L. (1998). DNA Directed Synthesis of Binary Nanoparticle Network Materials. Journal of America Chemical Society, 120, 12674-12675. https://doi.org/10. $1021 / \mathrm{ja} 982721 \mathrm{~s}$

Nuzzo, R. G., \& Allara, D. L. (1983).Adsorption of Bifunctional Organic Disulfides on Gold Surfaces. Journal of American Chemical Society, 105, 4481-4483. https://doi.org/10.1021/ja00351a063

Pearson, A. C., Liu, J., Pound, E., Uprety, B., Woolley, A. T., Davis, R. C., \& Harb, J. N. (2012).DNA Origami Metallized Site Specifically to Form Electrically Conductive Nanowires. Journal of Physical Chemistry, B 116, 10551-10560. https://doi.org/10.1021/jp302316p

Rothemund, P. (2006). Folding DNA to create nanoscale shapes and patterns. Nature, 440, 297-302. https://doi.org/10.1038/nature04586

Seeman, N. C. (1982). Nucleic acid junctions and lattices. Journal of Theoretical Biology, 99, 237-247. https://doi.org/10.1016/0022-5193(82)90002-9

Shen, X., Song, C., Wang, J., Shi, D., Wang, Z., Liu, N., \& Ding, B. (2012). Rolling up gold nanoparticle-dressed DNA origami into three dimensional plasmonicChiral nanostructures. Journal of America Chemical Society, 134, 146-149. https://doi.org/10.1021/ja209861x

Storhoff, J. J., Lazarides, A. A., Mucic, R. C., Mirkin, C. A., Letsinger, R. L., \& Schatz, G. C. (2000). What controls the Optical Properties of DNA-Linked Gold Nanoparticle Assemblies? Journal of America Chemical Society, 122, 4640-4650. https://doi.org/10.1021/ja9938251

Storhoff, J. J., Elghanian, R., Mucic, R. C., Mirkin, C. A., \& Letsinger, R. L. (1998). One-Pot Colorimetric Differentiation of Polynucleotides with Single Base Imperfections Using Gold Nanoparticle Probes. Journal of America Chemical Society, 120, 1959-1964. https://doi.org/10.1021/ja972332i 
Tanton, T. A. (2002). Preparation of Gold Nanoparticle-DNA Conjugates. Current Protocols in Nucleic Acid Chemistry, 12.12.11-12.12.12. https://doi.org/10.1002/0471142700.nc1202s09

Yang, M., Yau, H. C. M., \& Chan, H. L. (1998).Adsorption Kinetics and Ligand-Binding Properties of ThiolModified Double-Stranded DNA on a Gold Surface. Langmuir, 14, 6121-6129. https://doi.org/10.1021/la98 $0577 \mathrm{i}$

Zhao, Z., Jacovetty, E. L., Liu, Y., \& Yan, H. (2011). Encapsulation of Gold Nanoparticles in a DNA Origami Cage.Angewandte Chemie International Edition, 50, 2041-2044. https://doi.org/10.1002/anie.201006818

\section{Copyrights}

Copyright for this article is retained by the author(s), with first publication rights granted to the journal.

This is an open-access article distributed under the terms and conditions of the Creative Commons Attribution license (http://creativecommons.org/licenses/by/4.0/). 